\title{
Entomophthoramycosis: An unusual cause of facial disfigurement
}

\author{
Agnibho Mondal', Ayan Basu',*, Madhuchchanda Mandal', Arijit Mallik', \\ Dolan Champa Modak ${ }^{1}$, Dipankar Pal ${ }^{1}$, Debajyoti Majumder ${ }^{1}$, Subhasish Kamal Guha ${ }^{1}$ \\ ${ }^{1}$ Department of Tropical Medicine, School of Tropical Medicine, Kolkata, India; \\ ${ }^{2}$ Department of Infectious Diseases \& Advanced Microbiology, School of Tropical Medicine, Kolkata, India.
}

\begin{abstract}
SUMMARY Entomophthoramycosis is a rare fungal infection of nose, paranasal sinuses and subcutaneous tissues found in tropical and subtropical region. From India very few cases have been reported. Here we report a case of Entomophthoramycosis due to Conidiobolus coronatus from the eastern India who presented with slowly growing rhinofacial swelling and right sided nasal obstruction due to intranasal mass. The case was diagnosed by typical histopathological findings of broad aseptate hyphae with surrounding eosinophilic granular material (Splendore Hoeppli phenomenon) on microscopy of nasal biopsy material and confirmed by PCR assay of DNA and sequencing from biopsy tissue. Treatment with saturated solution of potassium iodide and itraconazole was successful and clinical cure was attained in 8 months.
\end{abstract}

Keywords Entomophthoramycosis, Conidiobolus coronatus, Splendore Hoeppli phenomenon

Entomophthoramycosis is a rare fungal infection of tropical and subtropical region. The disease usually affects adult males involved in agricultural works (1). It is caused by the fungi of the order Entomophthorales under the class Zygomycetes. However, unlike order Mucorales (another zygomycosis), entomophthoramycosis occurs predominantly in immunocompetent patients, is non angioinvasive and has chronic course (2). It has two genera, Conidiobolus and Basidiobolus. The former usually involves the rhinofacial area whereas the latter mostly involves the subcutaneous structures of trunk, arms or the gastrointestinal tract (1). Here we present a case of rhinofacial entomophthoramycosis caused by Conidiobolus coronatus in an immunocompetent host from Eastern India.

A 55-year old man, farmer, resident of West Bengal, presented with gradually increasing swelling of nose and face and right sided nasal obstruction for last three months. Initially there was a small mass inside the right nasal cavity which gradually increases in size causing nasal obstruction. After few days there was gradually increasing painless swelling of the dorsum of the nose, forehead, bilateral cheeks and the upper lip. It caused significant disfigurement of the face (Figure 1). He also complained of two episodes of epistaxis. He did not give any history of trauma. He consulted several doctors but without any improvement he came to our outpatient department. On examination the rhinofacial swelling was firm to hard in consistency. There was mild tenderness over both the maxillary sinuses. All other physical examinations were within normal limits. Routine blood examinations like complete blood count, liver and kidney function tests, fasting blood sugar, $\mathrm{HbAlc}$, HIV showed no abnormality. Computed tomography of paranasal sinus showed polypoidal mucosal thickening in maxillary, ethmoid, sphenoid and frontal sinuses. Bilateral turbinates were hypertrophied. Diffuse soft tissue thickening was noted in right nasolabial area and over maxilla. Magnetic resonance imaging also confirmed these findings. It also showed an enhancing altered signal intensity lesion in mid portion and adjacent bilateral fronto-naso-ethmoidal-maxillary areas and upper lip, mildly extending into the nasal cavities. Endoscopic examination revealed polypoid growth in the right nasal cavity. Histopathology of the biopsy material showed epitheloid granulomas with foreign body giant cells in haematoxylin eosin (H\&E) stain (Figure 2A). Broad thin walled aseptate fungal hyphae were also seen by Periodic Acid Schiff stain (Figures 3A and 3B). Each hyphal filament was enveloped by eosinophilic granular material known as Splendore Hoeppli phenomenon (Figure 2B). No vascular involvement was noted. Though culture of the biopsy material in Sabouraud's dextrose agar media yielded no growth but based on the clinical presentation and typical histopathological findings we suspected the case as entomophthoramycosis probably caused by Conidiobolus. Later PCR assay of DNA from biopsy tissue and sequencing identified the 


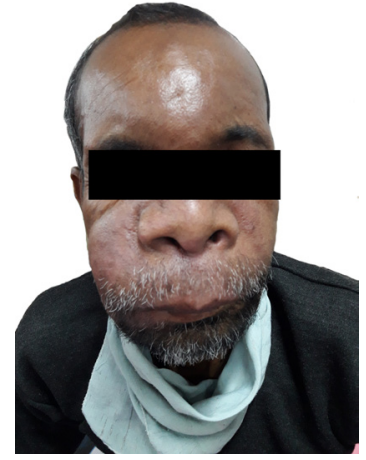

Figure 1. Rhinofacial lesion at the time of presentation.

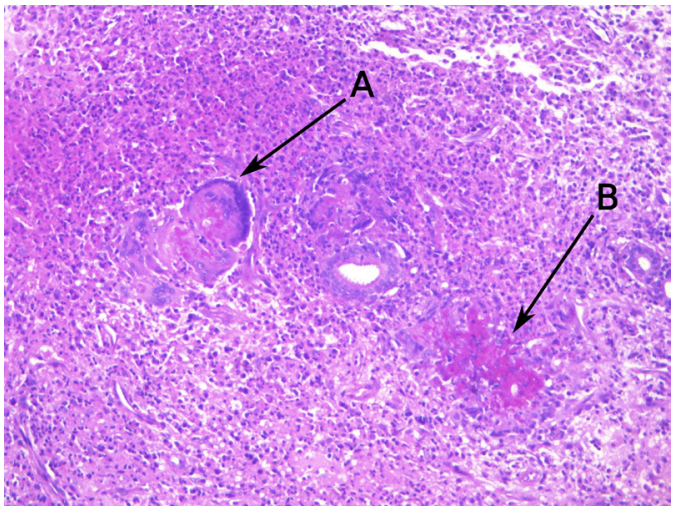

Figure 2. Nasal biopsy Histopathology stained by HaematoxylinEosin Stain with 20X magnification. A: Epitheloid granuloma with giant cell reaction; B: fungal filament enveloped by eosinophilic Splendore Hoeppli phenomenon.

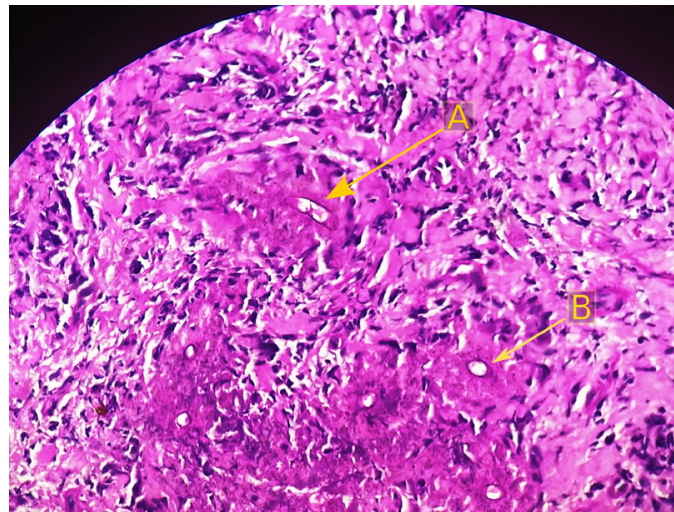

Figure 3. Nasal biopsy histopathology stained by Periodic Acid Schiff stain with $40 \mathrm{X}$ magnification showing fungal filaments.

fungus as Conidiobolus coronatus. We started treatment with itraconazole $200 \mathrm{mg}$ per capsule, one capsule thrice daily for 3 days then continued as twice daily dose and saturated solution of potassium iodide (SSKI) at a dose of 5 drops thrice daily. It was gradually increased up to 30 drops thrice daily. Each drop of freshly prepared SSKI contains approximately $65 \mathrm{mg}$ of potassium iodide. After one month the facial swelling reduced significantly and consistency became softer. After 8 months of therapy the lesions were dramatically improved.

Entomophthoramycosis is a chronic granulomatous subcutaneous infection which is acquired by inhalation or minor trauma. Conidiobolomycosis is mainly caused by
Conidiobolus coronatus whereas Basidiobolomycosis is caused by Basidiobolus ranarum (3). Conidiobolomycosis usually presents with unilateral nasal obstruction, nasal discharge, epistaxis, sinus tenderness and extensive facial swelling resulting in facial disfigurement. Conidiobolomycosis is diagnosed by characteristic rhinofacial swelling and typical histopathological findings of broad, aseptate or sparsely septated fungal hyphae surrounded by eosinophilic granular material known as Splendore Hoeppli phenomenon on microscopy of biopsy and confirmed by PCR assay and sequencing of DNA from biopsy tissue (3). Often culture is negative. During microscopy entomophthoramycosis must be differentiated from mucormycosis (4). Splendore Hoeppli phenomenon is very common in entomophthoramycosis but it is uncommon in mucormycosis. Vascular involvement is characteristic of mucormycosis whereas vessels are spared in entomophthoramycosis. Entomophthoramycosis occurs in immunocompetent individuals and has slow clinical course, whereas mucormycosis is seen in immunocompromised patients and has very rapid, aggressive course. Treatment options for entomophthoramycosis include SSKI, cotrimoxazole, amphotericin B and azole group of antifungals with varying clinical outcome and success. Currently combination of a SSKI and itraconazole appears to be the preferred drugs for rhinofacial conidiobolomycosis (5). The first case of entomophthoramycosis in humans was reported in 1965 (6). A review article on Conidiobolus showed that infection usually starts in the nasal mucosa of the inferior turbinate, then it gradually progresses to involve the dorsum of nose, forehead, cheeks and upper lip (7). The appearance of the patient has often been described as tapir or hippopotamus. Entomophthoramycosis is a rare disease and very few cases were reported from India $(8,9)$.

As cases of entomophthoramycosis are very rare it is easy to misdiagnosis a case. A very high index of clinical suspicion is essential to correctly diagnose a case of entomophthoramycosis in our clinical practice.

\section{Acknowledgements}

We thank Department of Tropical Medicine, School of Tropical Medicine, Kolkata, India.

Funding: None.

Conflict of Interest: The authors have no conflicts of interest to disclose.

\section{References}

1. Gugnani HC. Entomophthoromycosis due to Conidiobolus. Eur J Epidemiol. 1992; 8:391-396.

2. Elgart ML. Zygomycosis. Dermatol Clin. 1996; 14:141146.

3. Kontoyiannis DP, Lewis RE. Agents of Mucormycosis 
and Entomophthoramycosis. In: Principles and Practice of Infectious Diseases (Mandell, Douglas, Bennett, eds.). 9th Ed., Philadelphia, Elsevier, 2020; pp. 3117-3130.

4. Das P, Vijay MK, Joshi P, Yadav R, Singh G. Histological identification of entomophthoromycosis in biopsy samples is required. Indian J Pathol Microbiol. 2014; 57:514-516.

5. Gupta M, Narang T, Kaur RJ, Manhas A, Saikia UN, Dogra S. A prospective case series evaluating efficacy and safety of combination of itraconazole and potassium iodide in rhinofacial conidiobolomycosis. Int J Dermatol. 2016; 55:208-214.

6. Bras G, Gordon CC, Emmons CW, Prendegast KM, Sugar M. A case of phycomycosis observed in Jamaica; Infection with Entomophthora coronata. Am J Trop Med Hyg. 1965; 14:141-145.

7. Gugnani HC. Entomophthoromycosis due to Conidiobolus. Eur J Epidemiol. 1992; 8:391-396.
8. Mukhopadhyay D, Ghosh LM, Thammayya A, Sanyal M. Entomophthoromycosis caused by Conidiobolus coronatus: clinicomycological study of a case. Auris Nasus Larynx. 1995; 22:139-142.

9. Ramesh A, Deka RC, Vijayaraghavan M, Ray R, Kabra SK, Rakesh K, Manoj K. Entomophthoromycosis of the nose and paranasal sinus. Indian J Pediatr. 2000; 67:307310 .

Received October 30, 2021; Revised December 24, 2021; Accepted December 27, 2021.

\section{*Address correspondence to:}

Ayan Basu, Department of Infectious Diseases \& Advanced Microbiology, School of Tropical Medicine, 108, Chittaranjan Avenue, Kolkata- 700073, West Bengal, India.

E-mail: ayanbasustm@gmail.com 